\title{
Escherichia coli productora de betalactamasas de espectro extendido como agente causal de gangrena de Fournier de origen urogenital asociada a mayor mortalidad
}

\author{
Escherichia coli producer of extended-spectrum beta-lactamases as causative agent of \\ Fournier gangrene of urogenital origin associated with higher mortality
}

Luis D. Carrillo-Córdova*, Samuel Aguilar-Aizcorbe, Miguel A. Hernández-Farías, Christian Acevedo-García, Guillermo Soria-Fernández y Mateo L. Garduño-Arteaga

Departamento de Urología, Hospital General de México Dr. Eduardo Liceaga, Ciudad de México, México

\begin{abstract}
Resumen
Antecedentes: La gangrena de Fournier (GF) es una fascitis necrotizante que pone en peligro la vida del paciente. El objetivo de este trabajo fue determinar la etiología y el impacto del agente aislado en el cultivo de la herida y de orina. Método: Se llevó a cabo un análisis retrospectivo de una cohorte de 66 pacientes con GF de origen urogenital. Los valores cualitativos medidos se expresaron como frecuencia y porcentaje, y se compararon con la prueba de ji al cuadrado y la prueba de Fisher. La diferencia se consideró estadísticamente significativa con $p<0.05$. Resultados: Los pacientes que murieron presentaban con mayor frecuencia cultivos de orina y herida positivos para Escherichia coli productora de betalactamasas de espectro extendido (BLEE): orina, sobrevivientes $14.5 \%$ vs. muertes $44.4 \%$; herida, sobrevivientes $20.8 \%$ vs. muertes $66.6 \%(p<0.001)$. Conclusiones: Durante la valoración integral del paciente con GF es fundamental realizar cultivos de orina y de herida con el fin de iniciar el manejo antibiótico dirigido de manera temprana. Los pacientes con GF que mueren presentan mayor número de cultivos positivos para E. coli BLEE.
\end{abstract}

PALABRAS CLAVE: Gangrena de Fournier. Mortalidad. Infección de vías urinarias. Escherichia coli BLEE.

\begin{abstract}
Background: Fournier gangrene (FG) is a necrotizing fasciitis that endangers the patient's life. The objective of this study was to determine the etiology and impact of the agent isolated on wound and urine culture. Method: We performed a retrospective analysis within a cohort of 66 patients with FG of urogenital origin. The measured qualitative values were expressed as frequency and Percentage and compared with the chi square test and Fisher's test. The difference was considered statisticaIly significant at $p<0.05$. Results: Patients who died had more frequent cultures of urine and wound positive for extended-spectrum beta-lactamase (ESBL)-producing Escherichia coli: urine, survivors $14.5 \%$ vs. deaths $44.4 \%$; wound, $20.8 \%$ vs. $66.6 \%$ $(p<0.001)$. Conclusions: During the integral evaluation of the patient with FG it is essential to perform the urine and surgical wound cultures in order to initiate the antibiotic management directed at an early stage. Patients with GF who die present a greater number of cultures positive for $E$. coli ESBL.
\end{abstract}

KEY WORDS: Fournier gangrene. Mortality. Urinary tract infection. Escherichia coli ESBL.

\author{
Correspondencia: \\ *Luis D. Carrillo-Córdova \\ Cerro Teponaxtle 109 \\ Col. Campestre Churubusco, Del. Coyoacan \\ C.P. 04200 , Ciudad de México, México \\ E-mail: carrillocor@gmail.com
}

Fecha de recepción: 14-03-2018

Fecha de aceptación: 20-04-2018

DOI://dx.doi.org/10.24875/CIRU.M18000050
Cir Cir. 2018;86:327-331

Contents available at PubMed www.cirugiaycirujanos.com 


\section{Introducción}

La gangrena de Fournier (GF) es una fascitis necrotizante que pone en peligro la vida del paciente. Ocurre principalmente en pacientes con cierto grado de inmunosupresión ${ }^{1-5}$. Tiene una incidencia de 1.6 por 100,000 habitantes, y afecta principalmente a los hombres, aunque existen casos reportados en mujeres. Los principales factores de riesgo para presentarla son diabetes mellitus, obesidad, alcoholismo crónico, falla renal, insuficiencia hepática, tabaquismo, cáncer e infección por el virus de la inmunodeficiencia humana ${ }^{6}$. La mortalidad presentada en las primeras series era del $88 \%$, siendo en las más modernas del $7.5-16 \%{ }^{7,8}$. Los principales agentes causales son anaerobios (54\%), Escherichia coli (46.6\%) y estreptococos $(36.8 \%)^{8,9}$.

El objetivo de este trabajo fue determinar la etiología y el impacto del agente aislado en el cultivo de la herida y de orina en pacientes con diagnóstico de GF tratados en el Hospital General de México Dr. Eduardo Liceaga.

\section{Método}

La información de los pacientes necesaria para el análisis se recabó en el Hospital General de México Dr. Eduardo Liceaga. Se buscaron expedientes en el archivo clínico que coincidieran con este diagnóstico en el periodo comprendido de enero de 2011 a diciembre de 2017. Se evaluaron 120 expedientes de pacientes con diagnóstico de GF de origen urogenital como motivo de egreso. Se excluyeron 54 pacientes por no cumplir con los criterios diagnósticos (abscesos escrotales, abscesos inguinales, prostatitis agudas y crónicas). Se incluyeron 66 pacientes cuyo diagnóstico se realizó de manera clínica, fueron manejados por el servicio de urología, se les realizó un desbridamiento quirúrgico extenso y se les administró antibioticoterapia de amplio espectro.

Se llevó a cabo un análisis retrospectivo de esta cohorte de pacientes con GF de origen urológico. Las variables que se analizaron fueron el microorganismo que se desarrolló en el cultivo de orina y en el cultivo de herida, y la antibioticoterapia seleccionada, realizando una comparación entre el grupo de sobrevivientes $(n=48)$ y los que murieron $(n=18)$.Se evaluaron 66 pacientes, los cuales se incluyeron para el análisis estadístico.
El análisis estadístico se realizó utilizando el paquete estadístico para ciencias sociales (SPSS) para Windows versión 20.0.

Los valores cualitativos medidos se expresaron como frecuencia y porcentaje, y se compararon con la prueba de ji al cuadrado y la prueba de Fisher. La diferencia se consideró estadísticamente significativa con $p<0.05$, con un intervalo de confianza del $95 \%$.

\section{Resultados}

La población total consistió en 66 pacientes, de los que hubo 48 sobrevivientes y 18 muertes (27.7\%), con una edad promedio de 59.5 años, un peso promedio de $76.5 \mathrm{~kg}$ y un índice de masa corporal promedio de 27.7. En cuanto a la extensión de la fascitis necrotizante, el $28 \%$ presentó involucro pene-escrotal, el $53 \%$ perineal y el $16 \%$ inguino-abdominal. El $56.7 \%$ tenían antecedente de diagnóstico o cumplían criterios diagnósticos de diabetes mellitus tipo 2, y el $22.4 \%$ tenían antecedente de hipertensión. El etilismo fue positivo en el $44.8 \%$ de los pacientes.

En la biometría hemática predominó la leucocitosis con neutrofilia; se encontró un promedio de leucocitos de $23 / \mathrm{mm}^{3}$ (desviación estándar [DE]: 7.4 ), un $89 \%$ (DE: 6 ) de neutrófilos y un 5\% (DE: 2) de linfocitos. La cifra promedio de hemoglobina fue de 12.9 (DE: 3) y el $50 \%$ de los pacientes tenían anemia. El conteo plaquetario promedio fue de $254 / \mathrm{mm}^{3}$ (DE: 18). Al analizar la química sanguínea encontramos que la cifra promedio de glucosa fue de $253 \mathrm{mg} / \mathrm{dl}$ (DE: 50), la de creatinina $2.54 \mathrm{mg} / \mathrm{dl}$ (DE: 1.6), la de urea $124 \mathrm{mg} / \mathrm{dl}$ (DE: 68), la de sodio $131 \mathrm{mEq} / \mathrm{l}(\mathrm{DE}: 5)$, la de potasio $4.8 \mathrm{mEq} / \mathrm{l}$ (DE: 1), la de calcio $8 \mathrm{mEq} / \mathrm{l}$ (DE: 0.82), la de bicarbonato 16.8 (DE: 5), la de albúmina $1.9 \mathrm{~g} / \mathrm{dl}$ (DE: 0.5) y la de colesterol $114 \mathrm{U} / \mathrm{l}$ (DE: 26). El INR (International Normalized Ratio) fue en promedio 1.5 (DE: 1.6) (Tabla 1).

El urocultivo se encontró positivo en el $68.7 \%$ $(n=45)$. Se aisló Candida albicans en el $17.9 \%$ $(n=12)$ y gramnegativos en el $17.9 \%(n=12)$, seguidos de E. coli en el $13.4 \%(n=9)$ y de E. coli productora de betalactamasas de espectro extendido (BLEE) en el $9 \%(n=6)$; se halló un origen polimicrobiano en el $9 \%(n=6)$. Al realizar el análisis de los cultivos de la herida se encontró una mayor variedad de microorganismos. El principal agente aislado de la herida fue E. coli BLEE, la cual se encontró en el $32.8 \%(n=22)$ de los pacientes, seguida de $E$. coli y gramnegativos, cada uno con el $13.4 \%(n=9)$, el binomio $C$. albicans + E. Coli con el 6\% $(n=4)$, y Acinetobacter baumannii, 
Tabla 1. Descripción de las variables dentro de la población, en medias y porcentajes

\begin{tabular}{|c|c|c|c|}
\hline Variable & $\begin{array}{c}\text { Total } \\
(n=66)\end{array}$ & Variable & $\begin{array}{c}\text { Total } \\
(n=66)\end{array}$ \\
\hline Leucocitos $\left(\mathrm{mm}^{3}\right)$ & $23(7.4)$ & Diabetes (\%) & $56.7(38)$ \\
\hline Neutrófilos (\%) & $89(6)$ & Hipertensión (\%) & $22.4(15)$ \\
\hline Linfocitos (\%) & $5(2)$ & Etilismo (\%) & $44.8(30)$ \\
\hline $\begin{array}{l}\text { Hemoglobina } \\
\left(\mathrm{mm}^{3}\right)\end{array}$ & $12.9(3)$ & $\begin{array}{l}\text { Desencadenantes (\%) } \\
\text { Sonda>1 año } \\
\text { Orquiepididimitis } \\
\text { Litiasis uretral } \\
\text { Antecedente de RTUP } \\
\text { Estenosis de uretra }\end{array}$ & $\begin{array}{c}15(10) \\
13.6(9) \\
4.5(3) \\
4.5(3) \\
3(2)\end{array}$ \\
\hline $\begin{array}{l}\text { Hematocrito } \\
\left(\mathrm{mm}^{3}\right)\end{array}$ & $254(18)$ & $\begin{array}{l}\text { Factores de riesgo (\%) } \\
\text { ERC } \\
\text { Cirrosis } \\
\text { EPOC }\end{array}$ & $\begin{array}{l}15.5(10) \\
12.2(8) \\
7.5(5)\end{array}$ \\
\hline Plaquetas $\left(\mathrm{mm}^{3}\right)$ & $254(8)$ & IVU (\%) & $85(57)$ \\
\hline Glucosa (mg/dl) & $253(50)$ & $\begin{array}{l}\text { IPSS (\%) } \\
\text { Leve } \\
\text { Moderado } \\
\text { Grave }\end{array}$ & $\begin{array}{l}25.4(17) \\
55.2(37) \\
17.9(12)\end{array}$ \\
\hline $\begin{array}{l}\text { Creatinina } \\
(\mathrm{mg} / \mathrm{dl})\end{array}$ & $2.54(1.6)$ & Sepsis (\%) & $80.30(53)$ \\
\hline Urea (mg/dl) & $124(68)$ & Sepsis grave (\%) & $9(6)$ \\
\hline Sodio (mEq/l) & $131(5)$ & Talla suprapúbica (\%) & $58.2(39)$ \\
\hline Potasio (mEq/l) & $4.8(1)$ & $\begin{array}{l}\text { CONUT (\%) } \\
\text { Leve } \\
\text { Moderada } \\
\text { Grave }\end{array}$ & $\begin{array}{c}52.2(5) \\
37.3(25) \\
9 \%(6)\end{array}$ \\
\hline Calcio (mEq/l) & $8(82)$ & Ingreso a UCl & $35.8 \%(24)$ \\
\hline $\begin{array}{l}\text { Bicarbonato } \\
(\mathrm{mmol})\end{array}$ & $16.8(5)$ & Hemodiálisis & $22 \%(15)$ \\
\hline $\begin{array}{l}\text { Albúmina } \\
\text { (g/dl) }\end{array}$ & $1.9(.5)$ & INR & $1.5(1.6)$ \\
\hline Colesterol (U/I) & $114(26)$ & IMC (DE) & $27.7(6)$ \\
\hline
\end{tabular}

DE: desviación estándar; ERC: enfermedad renal crónica; EPOC: enfermedad pulmonar obstructiva crónica; IMC: índice de masa corporal; INR: international normalized ratio; IVU: infección de vías urinarias; IPSS: international prostate symptom score; RTUP: resección transuretral de próstata; UCl: unidad de cuidados intensivos

C. albicans, Enterococcus faecalis, Proteus mirabilis, Pseudomonas aeruginosa, Staphylococcus hominis y estafilococos hemolíticos cada uno con el $4.5 \%$ $(n=3)$. Cuatro pacientes no presentaron desarrollo en el cultivo de herida. En la tabla 2 se muestra el comparativo de los microorganismos aislados en orina y sangre entre los grupos de sobrevivientes y fallecidos. Al realizar el análisis estadístico se encontró que los pacientes que murieron presentaban con mayor frecuencia cultivos de orina y herida positivos para E. coli BLEE: orina, sobrevivientes $14.5 \%$ vs. muertes $44.4 \%$; herida, $20.8 \%$ sobrevivientes vs. $66.6 \%$ muertes $(p<0.001)$.

El manejo antibiótico fue a base de ertapenem en el $47.6 \%$ de los casos ( $n=32$ ), imipenem en el $17.9 \%$ $(n=12)$, imipenem con vancomicina en el $14.9 \%$ $(n=10)$, ertapenem con vancomicina en el $9 \%(n=6)$, y piperacilina-tazobactam y vancomicina en el $9 \%$ $(n=6)$.

\section{Discusión}

Existen muchos estudios en la literatura que han buscado encontrar los factores asociados a una mortalidad mayor en los pacientes con diagnóstico de GF, pero ninguno ha analizado el impacto de la microbiología en la evolución de la enfermedad. Se ha encontrado que someter a los pacientes a terapia de vacío disminuye significativamente la mortalidad ${ }^{9}$. Otro factor asociado a mayor mortalidad es el porcentaje de superficie corporal afectada, principalmente cuando esta es mayor del $3.25 \%{ }^{10}$.

Valores anormales, tanto clínicos como de laboratorio, incluyendo frecuencia cardiaca, temperatura, presión sanguínea, frecuencia respiratoria, conteo leucocitario, urea sérica, creatinina sérica, bicarbonato sérico, lactato sérico, calcio sérico, sodio sérico, potasio sérico y albumina sérica, se han asociado a mortalidad en la GF ${ }^{11-15}$.

En cuanto al microorganismo aislado en los pacientes con GF, existe un gran número de trabajos que han reportado sus hallazgos. Yilmazlar, et al..$^{16}$ encontraron que el agente más comúnmente aislado en sus pacientes fue E. coli (64\%), lo cual es muy similar a los hallazgos de este trabajo, en el que $E$. coli fue el microorganismo más aislado (26.8\%).

En los años 1980 se pensaba que la fascitis necrotizante era una infección causada por estreptococos, pero posteriormente se vio una mayor relación entre un origen polimicrobiano y la GF. Se ha mostrado que los pacientes con GF presentan un promedio de cuatro microorganismos distintos en el cultivo, siendo los más comunes estreptococos, estafilococos E. coli y $P$. aeruginosa, lo cual es muy similar a lo reportado en este trabajo (estafilococos $9 \%$, E. coli $26.8 \%$ y $P$. aeruginosa $4.5 \%)$; no se encontraron casos aislados de estreptococos. Nuestro laboratorio de microbiología no reportó más de una cepa bacteriana en cada cultivo $0^{17-19}$. 
Tabla 2. Microorganismos aislados en los cultivos de orina y de herida en pacientes con gangrena de Fournier y comparación entre sobrevivientes y muertos

\begin{tabular}{|c|c|c|c|c|c|c|}
\hline Cultivo positivo & $\begin{array}{l}\text { Orina (\%) } \\
(n=66)\end{array}$ & $\begin{array}{l}\text { SV (\%) } \\
(n=48)\end{array}$ & $\begin{array}{l}M(\%) \\
(n=18)\end{array}$ & $\begin{array}{l}\text { Herida (\%) } \\
(n=66)\end{array}$ & $\begin{array}{l}\text { SV (\%) } \\
(n=48)\end{array}$ & $\begin{array}{l}M(\%) \\
(n=18)\end{array}$ \\
\hline C. albicans & $\begin{array}{l}17.9 \\
(n=12)\end{array}$ & $\begin{array}{l}16.6 \\
(n=8)\end{array}$ & $\begin{array}{l}22.2 \\
(n=4)\end{array}$ & 0 & 0 & 0 \\
\hline Gramnegativos & $\begin{array}{l}17.9 \\
(n=12)\end{array}$ & $\begin{array}{l}20.8 \\
(n=10)\end{array}$ & $\begin{array}{l}11.1 \\
(n=2)\end{array}$ & $\begin{array}{l}13.4 \\
(n=9)\end{array}$ & $\begin{array}{l}16.6 \\
(n=8)\end{array}$ & $\begin{array}{l}5.5 \\
(n=1)\end{array}$ \\
\hline E. coli & $\begin{array}{l}13.4 \\
(n=9)\end{array}$ & $\begin{array}{l}14.5 \\
(n=7)\end{array}$ & $\begin{array}{l}11.1 \\
(n=2)\end{array}$ & $\begin{array}{l}13.4 \\
(n=9)\end{array}$ & $\begin{array}{l}16.6 \\
(n=8)\end{array}$ & $\begin{array}{l}5.5 \\
(n=1)\end{array}$ \\
\hline E. coli BLEE & $\begin{array}{l}13.4 \\
(n=9)^{*}\end{array}$ & $\begin{array}{l}2 \\
(n=1)\end{array}$ & $\begin{array}{l}44.4 \\
(n=8)\end{array}$ & $\begin{array}{l}32.8 \\
(n=22)^{*}\end{array}$ & $\begin{array}{l}20.83 \\
(n=10)\end{array}$ & $\begin{array}{l}66.6 \\
(n=12)\end{array}$ \\
\hline Polimicrobiano & $\begin{array}{l}9 \\
(n=6)\end{array}$ & $\begin{array}{l}8.3 \\
(n=4)\end{array}$ & $\begin{array}{l}11.1 \\
(n=2)\end{array}$ & 0 & 0 & 0 \\
\hline E. coli+C. albicans & 0 & 0 & 0 & $\begin{array}{l}6 \\
(n=4)\end{array}$ & $\begin{array}{l}6.25 \\
(n=3)\end{array}$ & $\begin{array}{l}5.5 \\
(n=1)\end{array}$ \\
\hline A. baumannii & 0 & 0 & 0 & $4.5(n=3)$ & $\begin{array}{l}6.25 \\
(n=3)\end{array}$ & 0 \\
\hline E. faecalis & 0 & 0 & 0 & $\begin{array}{l}4.5 \\
(n=3)\end{array}$ & $\begin{array}{l}6.25 \\
(n=3)\end{array}$ & 0 \\
\hline P. mirabilis & 0 & 0 & 0 & $\begin{array}{l}4.5 \\
(n=3)\end{array}$ & $\begin{array}{l}6.25 \\
(n=2)\end{array}$ & $\begin{array}{l}5.5 \\
(n=1)\end{array}$ \\
\hline$P$. aeruginosa & 0 & 0 & 0 & $\begin{array}{l}4.5 \\
(n=3)\end{array}$ & $\begin{array}{l}2.08 \\
(n=1)\end{array}$ & $\begin{array}{l}11.1 \\
(n=2)\end{array}$ \\
\hline S. hominis & 0 & 0 & 0 & $\begin{array}{l}4.5 \\
(n=3)\end{array}$ & $\begin{array}{l}6.25 \\
(n=3)\end{array}$ & 0 \\
\hline Estafilococos hemolíticos & 0 & 0 & 0 & $\begin{array}{l}4.5 \\
(n=3)\end{array}$ & $\begin{array}{l}6.25 \\
(n=3)\end{array}$ & 0 \\
\hline Sin desarrollo & $\begin{array}{l}27.2 \\
(n=18)\end{array}$ & $\begin{array}{l}37.5 \\
(n=18)\end{array}$ & 0 & $\begin{array}{l}6.6 \\
(n=4)\end{array}$ & $\begin{array}{l}8.3 \\
(n=4)\end{array}$ & 0 \\
\hline
\end{tabular}

*Estadísticamente significativo $(p<0.05)$.

M: muertos; SV: sobrevivientes

Un estudio de una serie de 43 pacientes reportó que los microorganismos bacterianos más comunes encontrados en las heridas fueron $E$. coli $(48.8 \%)$, Pseudomonas spp. (20.9\%), Enterococcus spp. (18.6\%), Staphylococcus spp. (13.9\%), Streptococcus spp. (11.6\%), Proteus spp. (11.6\%), Acinetobacter spp. (9.3\%), Bacteroides spp. (9.3\%) y Klebsiella pneumoniae (4.6\%); no se realizó un análisis de la relación entre la morbimortalidad y el agente aislado en el cultivo $^{20}$.

Las bacterias aisladas de pacientes con GF suelen representar la flora normal de la región urogenital o anorrectal, tales como cepas entéricas (E. coli, KlebsieIla spp., Proteus spp.), cocos grampositivos (estafilococos, estreptococos, enterococos) y bacterias anaerobias (Clostridium spp., Bacteroides spp., Fusobacterium) $)^{21,22}$. Paty y Smith ${ }^{23}$ informaron de E. coli, Bacteroides spp. y estreptococos como las bacterias más comúnmente aisladas. Palmer, et al. ${ }^{24}$ reportaron que predominaban las cepas de E. coli y estreptococos, mientras que las cepas de Bacteroides spp. fueron cultivadas menos comúnmente en muestras de pacientes con GF. Ulug, et al. ${ }^{25}$ encontraron E. coli y $P$. aeruginosa como las bacterias más comúnmente aisladas de pacientes con GF. Ayan, et al. ${ }^{26}$ encontraron E. coli (58\%) y Staphylococcus aureus (36\%) como los agentes etiológicos más comunes de FG. En un estudio ${ }^{27}$ que incluyó 15 casos, los bacilos gramnegativos, E. coli y Acinetobacter spp. fueron los más comunes.

Recientemente, $S$. aureus resistente a la meticilina adquirido en la comunidad ha surgido como causa de GF con un curso clínico grave e incluso fulminante ${ }^{27}$. La mala higiene y el traumatismo local predisponen a desarrollar GF, ya que las bacterias tienen acceso a 
los tejidos más profundos. Se afirma que la sinergia entre bacterias anaerobias contribuye a la patogénesis de la GF. Estas bacterias secretan múltiples toxinas y enzimas que hacen que el tejido se necrose rápidamente (p. ej., hialuronidasa, estreptocinasa, colagenasa); además, predisponen a la formación de trombos en los vasos sanguíneos y a la insuficiencia cardiovascular grave ${ }^{28,29}$.

En nuestro trabajo se encontró una gran cantidad de pacientes con GF con cultivos positivos para $E$. coli BLEE, lo cual es un hallazgo que no se ha reportado anteriormente en la literatura. Al realizar la comparación entre los pacientes que sobrevivieron y los que murieron, estos últimos tenían un mayor número de cultivos positivos para este microorganismo, siendo estadísticamente significativo.

\section{Conclusión}

Durante la valoración integral del paciente con GF es fundamental realizar cultivos de orina y de herida quirúrgica con el fin de iniciar el manejo antibiótico dirigido de manera temprana. Los pacientes con GF que mueren presentan mayor número de cultivos positivos para E. coli BLEE.

\section{Conflicto de intereses}

Los autores de este trabajo no tienen ningún conflicto de intereses.

\section{Bibliografía}

1. Corcoran AT, Smaldone MC, Gibbons EP, Walsh TJ, Davies BJ. Validation of the Fournier's gangrene severity index in a large contemporary series. J Urol. 2008;180):944-8.

2. Ozturk E, Ozguc H, Yilmazlar T. The use of vacuum assisted closure therapy in the management of Fournier's gangrene. Am J Surg. 2009;197:660-5.

3. Czymek R, Schmidt A, Eckmann C, Bouchard R, Wulff B, Laubert T, et al. Fournier's gangrene: vacuum-assisted closure versus conventional dressings. Am J Surg. 2009;197:168-76.

4. Morua AG, López JA, García JD, Montelongo RM, Guerra LS. Fournier's gangrene: our experience in 5 years, bibliographic review and assessment of the Fournier's gangrene severity index. Arch Esp Urol. 2009;62:532-40.

5. Sroczyński M, Sebastian M, Rudnicki J, Sebastian A, Agrawal AK. A complex approach to the treatment of Fournier's gangrene. Adv Clin Exp Med. 2013;22:131-5.
6. Sorensen MD, Krieger JN. Fournier's gangrene: epidemiology and outcomes in the general US population. Urol Int. 2016;97:249-59.

7. Hagedorn JC, Wessells H. A contemporary update on Fournier's gangrene. Nat Rev Urol. 2017;14:205-14.

8. Tang LM, Su YJ, Lai YC. The evaluation of microbiology and prognosis of Fournier's gangrene in past five years. Springerplus. 2015;4:14.

9. Horsanali MO, Eser U, Horsanali BO, Altas O, Eren H. WITHDRAWN: comparison of vacuum-assisted closure therapy and debridement with primer surgical closure for Fournier's gangrene treatment: 10 years' experience of a single centre. Int Braz J Urol. 2017;43.

10. Morais $H$, Neves J, Maciel Ribeiro H, Ferreira M, Guimaraes N, Azenha $\mathrm{N}$, et al. Case series of Fournier's gangrene: affected body surface area - the underestimated prognostic factor. Ann Med Surg (Lond). 2017;16:19-22.

11. Lin TY, Ou CH, Tzai TS, Tong YC, Chang CC, Cheng HL, et al. Validation and simplification of Fournier's gangrene severity index. Int J Urol. 2014;21:696- 701.

12. Altarac S, Katusin D, Crnica S, Papes D, Rajkovic Z, Arslani F. Fournier's gangrene: etiology and outcome analysis of 41 patients. Urol Int. 2012;88:289- 93.

13. Ulug M, Gedik E, Girgin S, Celen MK, Ataz C. The evaluation of microbiology and Fournier's gangrene severity index in 27 patients. Int $\mathrm{J}$ Infect Dis. 2009;13:e424-30.

14. Kabay S, Yucel M, Yaylak F, Algin MC, Hacioglu A, Kabay B, et al. The clinical features of Fournier's gangrene and the predictivity of the Fournier's Gangrene Severity Index on the outcomes. Int Urol Nephrol. 2008;40:997-1004.

15. Erol B, Tuncel A, Hanci V, Tokgoz H, Yildiz A, Akduman B, et al. Fournier's gangrene: overview of prognostic factors and definition of new prognostic parameter. Urology. 2010;75:1193-8.

16. Yilmazlar T, Gulcu B, Isik O, Ozturk E. Microbiological aspects of Fournier's gangrene. Int J Surgery (Lond). 2017;40:135-8.

17. Meleney FL. Hemolytic streptococcus gangrene. Arch Surg. 1924;9: 317-364.

18. Ulug M, Gedik E, Girgin S, Celen MK, Ayaz C, et al. The evaluation of microbiology and Fournier's gangrene severity index in 27 patients. Int $\mathrm{J}$ Infect Dis. 2009;13:e424-30.

19. Singh A, Ahmed K, Aydin A, Khan MS, Dasgupta P. Fournier's gangrene. A clinical review. Arch Ital Urol. 2016;88:157-64.

20. Oguz A, Gumus M, Turkoglu A, Bozdag Z, Ulger BV, Agacayak E, et al. Fournier's gangrene: a summary of 10 years of clinical experience. Int Surg. 2015;100:934-41.

21. Eke N, Raphael JE. Fournier's gangrene. En: Vitin A, editor. Gangrene - current concepts and management options. InTech. 2011. p. 37-48. Disponible en: http://cdn.intechopen.com/pdfs/18914/InTech-Fournier_s_ gangrene.pdf.

22. European Association of Urology, National Guideline Clearinghouse (NGC). Fournier's gangrene. En: Guidelines on urological infections. Disponible en: http://www.guideline.gov/content.aspx?id=34103.

23. Paty R, Smith AD. Gangrene and Fournier's gangrene. Urol Clin North Am. 1992;19:149-62.

24. Palmer LS, Winter HI, Tolia BM, Reid RE, Laor E. The limited impact of involved surface area and surgical debridement on survival in Fournier's gangrene. Br J Urol. 1995;76:208-12.

25. Ulug M, Gedik E, Girgin S, Celen M, Ayaz C. The evaluation of microbiology and Fournier's gangrene severity index in 27 patients. Int $\mathrm{J}$ Infect Dis. 2009;13:e424-30.

26. Ayan F, Sunamak O, Paksoy SM, Polat SS, As A, Sakoglu N, et al. Fournier's gangrene: a retrospective clinical study on forty-one patients. ANZ J Surg. 2005;75:1055-8.

27. Burton MJ, Shah P, Swiatlo E. Community-acquired methicillin-resistant Staphylococcus aureus as a cause of Fournier's gangrene. Am J Med Sci. 2008;335:327-8.

28. Champion SE. A case of Fournier's gangrene: signs and symptoms. Urol Nurs. 2007;27:296-9.

29. Ersay A, Yilmaz G, Akgun Y, Celik Y. Factors affecting mortality of Fournier's gangrene: review of 70 patients. ANZ J Surg. 2007;77:
43-48. 